\title{
IDEMPOTENTS OF NORM ONE AND BANACH ALGEBRA REPRESENTATIONS OF COMPACT GROUPS
}

\author{
N. J. KALTON AND G. V. WOOD
}

\begin{abstract}
Let $G$ be a finite group of order $n$ and let $A$ be a (real or complex) Banach algebra. Rudin and Schneider [3] ask whether a mapping $f: G \rightarrow A$ satisfying $\|f(x)\|=1$ and $f(x)=(1 / n) \sum_{y \in G} f\left(x y^{-1}\right) f(y)$ is necessarily a homomorphism (Question 1, p. 602). They give an affirmative answer if $A$ is either commutative and semisimple or strictly convex.

Here, we prove this result for general Banach algebras, and at the same time prove the natural generalization to compact groups. This allows us to characterize norm one idempotents in generalized group algebras.
\end{abstract}

Suppose that $G$ is a compact group with identity $e$ and that $X$ is a normed space. A representation of $G$ on $X$ is a homomorphism $T: G \rightarrow B(X)$. $T$ is an isometric representation if in addition each $T_{x}$ is an isometry on $X$; in this case $T_{e}=I$ and each $T_{x}$ is invertible. $T$ is semi-isometric if $\left\|T_{x}\right\| \leqslant 1$ for $x \in G$; in this case $T_{e}$ is a projection of norm one and $T_{x}=S_{x} T_{e}$ where $S$ is an isometric representation of $G$ on $T_{e}(X)$.

We equip $G$ with its left-invariant Haar measure $\lambda$, normalized so that $\lambda(G)=1$; we shall abbreviate $d \lambda(x)$ to $d x$. If $X$ is any Banach space (or Banach algebra) then a map $\phi: G \rightarrow X$ is Bochner measurable if it is the almost everywhere limit of a sequence of simple functions, and Bochner integrable if in addition $\int\|\phi(x)\| d x<\infty$. An operator valued function $T$ : $G \mapsto B(X)$ is strongly measurable if for each $\xi \in X$, the map $x \rightarrow T_{x} \xi$ is Bochner measurable (see Hille-Phillips [2, pp. 72-74]). If for each $\xi \in X, T_{x} \xi$ is Bochner integrable and $T \xi=\int_{G} T_{x} \xi d x$, then we shall write $T=\int_{G} T_{x} d x$.

LEMMA 1. If $x \rightarrow T_{x}$ is a strongly measurable mapping from $G$ into $B(X)$, then so is the map $y \mapsto T_{x y-1} T_{y}$.

We omit the proof of Lemma 1, which follows by approximation by simple functions.

Lemma 2. If $\phi: G \rightarrow X$ is Bochner integrable then

$$
\lim _{x \rightarrow e} \int_{G}\|\phi(u x-\phi(u))\| d u=0 .
$$

Proof. See [2, Theorem 3.8.3] for the case $G=\mathbf{R}^{n}$; the same proof applies here. The lemma is proved first for simple functions and follows in general by approximation. Again we omit the details.

Received by the editors August 6, 1975 .

AMS (MOS) subject classifications (1970). Primary 43A20; Secondary 43A65.

(c) American Mathematical Society 1976 
Lemma 3. Suppose $x \rightarrow T_{x}$ is a strongly measurable mapping from $G$ into $B(X)$ such that $\sup _{x \in G}\left\|T_{x}\right\|=M<\infty$. Suppose for $x \in G$,

$$
T_{x}=\int_{G} T_{x y^{-1}} T_{y} d y .
$$

Then $x \mapsto T_{x}$ is strongly continuous (i.e. for $\xi \in X, x \rightarrow T_{x} \xi$ is continuous).

Proof. For $\xi \in X$,

$$
\begin{aligned}
T_{x} \xi & =\int_{G} T_{x y^{-1}} T_{y} \xi d y, \\
T_{x u} \xi & =\int_{G} T_{x u y^{-1}} T_{y} \xi d y=\int_{G} T_{x y^{-1}} T_{y u} \xi d y,
\end{aligned}
$$

by the invariance of Haar measure. Hence

$$
\begin{aligned}
\left\|T_{x} \xi-T_{x u} \xi\right\| & \leqslant \int_{G}\left\|T_{x y^{-1}}\left(T_{y} \xi-T_{y u} \xi\right)\right\| d y \\
& \leqslant M \int_{G}\left\|T_{y} \xi-T_{y u} \xi\right\| d y \rightarrow 0 \quad \text { as } u \rightarrow e \text { by Lemma } 2 .
\end{aligned}
$$

LEMMA 4. If $x \mapsto T_{x}$ is a strongly continuous map from $G$ into $B(X)$ then

(i) $(x, y) \rightarrow T_{x} T_{y}$ is strongly continuous on $G \times G$,

(ii) $(x, y) \rightarrow T_{x}^{*} T_{y}^{*}$ is weak ${ }^{*}$-continuous on $G \times G$.

Proof. Since $G$ is compact, the continuous function $x \rightarrow\left\|T_{x} \xi\right\|$ is bounded for every $\xi \in X$. Hence the Uniform Boundedness Theorem shows that $\sup _{x \in G}\left\|T_{x}\right\|=M<\infty$. Then

$$
\begin{aligned}
\left\|T_{x} T_{y} \xi-T_{x_{0}} T_{y_{0}} \xi\right\| & \leqslant\left\|T_{x} T_{y} \xi-T_{x} T_{y_{0}} \xi\right\|+\left\|T_{x} T_{y_{0}} \xi-T_{x_{0}} T_{y_{0}} \xi\right\| \\
& \leqslant M\left\|T_{y} \xi-T_{y_{0}} \xi\right\|+\left\|\left(T_{x}-T_{x_{0}}\right)\left(T_{y_{0}} \xi\right)\right\| \\
& \rightarrow 0 \text { as } x \rightarrow x_{0} \text { and } y \rightarrow y_{0} .
\end{aligned}
$$

(ii) follows immediately by duality.

REMARK. It is not true that $x \mapsto T_{x}^{*}$ is strongly continuous from $G$ into $B\left(X^{*}\right)$. For example let $G=\prod_{i=1}^{\infty}\{-1,+1\}$ and consider the representation on $l_{1}$ given by $\left(T_{x} \xi\right)_{n}=x_{n} \xi_{n}$ for $\xi=\left(\xi_{n}\right) \in l_{1}$ and $x=\left(x_{n}\right) \in G$.

LEMma 5. Suppose $T: G \rightarrow B(X)$ is strongly continuous and satisfies

$$
\text { (i) }\left\|T_{x}\right\| \leqslant 1, \quad x \in G, \quad \text { (ii) } T_{x}=\int_{G} T_{x y^{-1}} T_{y} d y, \quad x \in G .
$$

Then, for $\xi \in X,\left\|T_{x} \xi\right\|$ is independent of $x$ and

$$
\left\|T_{x} \xi\right\|=\left\|T_{y} T_{z} \xi\right\| \quad \text { whenever } x, y, z \in G \text {. }
$$

Proof.

$$
\begin{aligned}
\left\|T_{x} \xi\right\| & \leqslant \int_{G}\left\|T_{x y^{-1}} T_{y} \xi\right\| d y \\
& \leqslant \int_{G}\left\|T_{y} \xi\right\| d y \quad \text { for any } x \in G .
\end{aligned}
$$

Hence $\left\|T_{x} \xi\right\|=\int_{G}\left\|T_{y} \xi\right\| d y$ for almost every $x \in G$. Strong continuity of $T_{x}$ ensures that equality holds everywhere. Referring back to inequality (1) we see that $\left\|T_{x} \xi\right\|=\left\|T_{x y-1} T_{y} \xi\right\|$ for almost every $y \in G$. Again by continuity 
equality holds everywhere and the result follows.

We are now able to prove the first version of our main result.

THEOREM 1. Suppose $T: G \rightarrow B(X)$ is strongly measurable and satisfies

(i) $\left\|T_{x}\right\| \leqslant 1(x \in G)$,

(ii) $T_{e}$ is an isometry, i.e. $\left\|T_{e} \xi\right\|=\|\xi\|$ for $\xi \in X$,

(iii) $T_{x}=\int_{G} T_{x y^{-1}} T_{y} d y(x \in G)$.

Then $T$ is a strongly continuous isometric representation of $G$.

Proof. $T$ is strongly continuous by Lemma 3 . Let $U$ be the closed unit ball of $X^{*}$ and $\phi$ be any extreme point of $U$. Since $T_{e}$ is an isometry, it follows by the Hahn-Banach Theorem that there exists $\psi \in U$ such that $T_{e}^{*} \psi=\phi$.

For $\xi \in X$,

$$
\phi(\xi)=\psi\left(T_{e} \xi\right)=\int_{G} \psi\left(T_{x} T_{x^{-1}} \xi\right) d x .
$$

For each measurable subset $A$ of $G$ with $\lambda(A)>0$ define $\phi_{A} \in X^{*}$ by

$$
\phi_{A}(\xi)=\lambda(A)^{-1} \int_{A} \psi\left(T_{x} T_{x^{-1}} \xi\right) d x .
$$

Clearly $\phi_{A} \in U$ and $\phi=\lambda(A) \phi_{A}+\lambda(G-A) \phi_{G-A}$. As $\phi$ is an extreme point of $U, \phi=\phi_{A}=\phi_{G-A}$. Thus

$$
\int_{A}\left(\phi(\xi)-\psi\left(T_{x} T_{x-1} \xi\right)\right) d x=0
$$

for every measurable $A \subset G$ and $\xi \in X$. Hence for $\xi \in X, \psi\left(T_{x} T_{x^{-1}} \xi\right)=\phi(\xi)$ almost everywhere, and by the strong continuity of the map $x \mapsto T_{x} T_{x^{-1}}$ (see Lemma 4), equality holds everywhere. Hence $T_{x-1}^{*} T_{x}^{*} \psi=\phi$ for $x \in G$. The choice of $\psi$ shows that $T_{e}^{*} \phi=\left(T_{e}^{*}\right)^{2} \psi=\phi$. As $T_{e}^{*}$ is weak*-continuous and, by the Krein-Milman theorem, $U$ is the weak*-closed convex cover of its extreme points we have $T_{e}^{*}=I$. Thus $\phi=\psi$ and we have also proved that $T_{x^{-1}}^{*} T_{x}^{*} \phi=\phi$ for any extreme point $\phi$, i.e. $T_{x} T_{x^{-1}}=I$ by the same argument as above. Hence each $T_{x}$ is an isometric isomorphism of $X$.

Again if $\phi$ is any extreme point of $U$, so is $T_{x}^{*} \phi$ and

$$
\left(T_{x}^{*} \phi\right)(\xi)=\int_{G} \phi\left(T_{x y-1} T_{y} \xi\right) d y \quad(\xi \in X) .
$$

Arguing as before we conclude that

$$
T_{x}^{*}=T_{y}^{*} T_{x y^{-1}}^{*}, \quad x, y \in G,
$$

i.e. $T$ is an isometric representation.

TheOREM 2. Suppose $T: G \rightarrow B(X)$ is strongly measurable and satisfies

(i) $\left\|T_{x}\right\| \leqslant 1(x \in G)$, $G$.

(ii) $T_{x}=\int T_{x y-1} T_{y} d y(x \in G)$.Then $T$ is a semi-isometric representation of

Proof. Again we have $T$ strongly continuous by Lemma 2. Define a seminorm $|\cdot|$ on $X$ by

$$
|\xi|=\left\|T_{e} \xi\right\|
$$

and let $N=T_{e}^{-1}(0)$. By Lemma $5,\left|T_{x} \xi\right|=|\xi|$ for $x \in G$. Hence there is an induced representation on $X / N$ satisfying the hypotheses of Theorem 1. By 
Theorem 1,

$$
\left|T_{e} \xi-\xi\right|=0, \quad \xi \in X
$$

and

$$
\left|T_{x} T_{y} \xi-T_{x y} \xi\right|=0, \quad \xi \in X, x, y \in G .
$$

Hence $\left\|T_{e}^{2} \xi-T_{e} \xi\right\|=0$, i.e. $T_{e}$ is a projection. By Lemma $5,\left\|T_{x}\left(T_{e} \xi-\xi\right)\right\|$ $=\left\|T_{e}\left(T_{e} \xi-\xi\right)\right\|=\left\|T_{e}^{2} \xi-T_{e} \xi\right\|=0$ for $x \in G$, i.e. $T_{x} T_{e}=T_{x}$. Also by Lemma 5 , for any $w \in G$,

$$
\left\|T_{w} T_{x} T_{y} \xi-T_{w} T_{x y} \xi\right\|=\left\|T_{e}\left(T_{x} T_{y} \xi-T_{x y} \xi\right)\right\|=\left|T_{x} T_{y} \xi-T_{x y} \xi\right|=0 .
$$

Thus $T_{w} T_{x} T_{y}=T_{w} T_{x y}$ for $w, x, y \in G$.

Now suppose we have the equation

$$
T_{e} T_{x}=T_{x} \quad(x \in G) .
$$

Then we have $T_{x} T_{y}=T_{e} T_{x} T_{y}=T_{e} T_{x y}=T_{x y}$, and the proof is complete. Therefore it remains only to establish (2). Here the only difficulty is that $x \rightarrow T_{x}^{*}$ need not be strongly continuous. (For, if it were, we could apply the argument above to $x \rightarrow T_{x}^{*}$.) This is circumvented by the construction that follows. We shall assume here that $X$ is complete, for convenience.

Fix any $\xi_{0} \in X$ and let $X_{0}$ be the smallest closed subspace of $X$ such that $\xi_{0} \in X_{0}$ and $T_{x}\left(X_{0}\right) \subset X_{0}, x \in G$. It is enough to consider the induced map $G \rightarrow B\left(X_{0}\right)$.

Let $C_{0}=\left\{\left(\xi_{0}\right) \cup\left(T_{x} \xi_{0}: x \in G\right) \cup\left(T_{x} T_{y} \xi_{0}: x, y \in G\right)\right\}$. Then $C_{0}$ is compact and so is its closed absolutely convex hull $C$. Let $Y$ be the linear span of $C$ equipped with the norm whose unit ball is $C$. Then $Y$ is a Banach space, since $C$ is compact. Furthermore since $T_{w} T_{x} T_{y}=T_{w} T_{x y}$ for $w, x, y \in G$ we have $T_{w}(C) \subset C$. Thus $Y$ is invariant for each $T_{x}$ and so $Y$ is dense in $X_{0}$. Let $\tilde{T}$ denote the restriction of $T_{x}$ to $Y$; then in the norm of $Y,\left\|\tilde{T}_{x}\right\| \leqslant 1$. Let $J$ : $Y \rightarrow X_{0}$ be the inclusion map. By construction $J$ is compact and $J \tilde{T}_{x}=T_{x} J$ $(x \in G)$. Now suppose $T_{e} T_{w} \neq T_{w}$. Since $T_{e}$ is a projection, $T_{w}\left(X_{0}\right)$ $\not \subset T_{e}\left(X_{0}\right)$. Hence there exists $\psi \in X_{0}^{*}$ such that $T_{w}^{*} \psi \neq 0$ but $T_{e}^{*} \psi=0$. Since $J$ is compact the map $x \rightarrow J^{*} T_{x}^{*} \psi$ is continuous. Choose $u \in G$ such that

$$
c=\left\|J^{*} T_{u}^{*} \psi\right\|=\max _{x \in G}\left\|J^{*} T_{x}^{*} \psi\right\| .
$$

Since $J(C)$ is compact there exists $\eta \in C$ such that $T_{u}^{*} \psi(J \eta)=c$. Then

$$
\left|\psi\left(T_{u x^{-1}} T_{x} J \eta\right)\right|=\left|\psi\left(T_{u x^{-1}} J \tilde{T}_{x} \eta\right)\right|=\left|J^{*} T_{u x^{-1}}^{*} \psi\left(\tilde{T}_{x} \eta\right)\right| \leqslant c .
$$

However

$$
\int_{G} \psi\left(T_{u x^{-1}} T_{x} J \eta\right) d x=\psi\left(T_{u} J \eta\right)=c .
$$

Hence $\psi\left(T_{u x^{-1}} T_{x} J \eta\right) \equiv c$ by continuity. In particular putting $x=u$, $\psi\left(T_{e} T_{u} J \eta\right)=c$, i.e. $T_{e}^{*} \psi\left(T_{u} J \eta\right)=c$. However $T_{e}^{*} \psi=0$ and hence $c=0$. Thus $J^{*} T_{w}^{*} \psi=0$; but $Y$ is dense in $X_{0}$ and hence $T_{w}^{*} \psi=0$, which is a contradiction to our initial assumption. This completes the proof.

Corollary. Suppose $\mathcal{Q}$ is a bounded subsemigroup of $B(X)$ and $T: G$ $\rightarrow B(X)$ is a strongly measurable mapping satisfying

(i) $T(G) \subset Q$, 
(ii) $T_{x}=\int_{G} T_{x y}{ }^{-1} T_{y} d y(x \in G)$.

Then $T$ is a representation of $G$.

Proof. Renorm $X$ by $|\xi|=\sup _{A \in \mathbb{Q} \cup\{I\}}\|A \xi\|$.

We can now characterize idempotents of norm one in the generalized group algebra of a locally compact group $G$. Let $A$ be a Banach algebra and let $L^{1}(G: A)$ denote the space of Bochner integrable functions $f: G \rightarrow A$. $L^{1}(G: A)$ is a Banach algebra under the multiplication

$$
f * g(x)=\int_{G} f\left(x y^{-1}\right) g(y) d y
$$

and norm

$$
\|f\|=\int_{G}\|f(x)\| d x .
$$

It is well known that if $A=C$, the norm one idempotents of $L^{1}(G: C)=L^{1}(G)$ are of the form $\lambda(H)^{-1} \rho(x) \chi_{H}(x)$ where $H$ is a compact open subgroup, $\rho$ is a character on $H$ and $\chi_{H}$ is the characteristic function of $H$. (See $[1,2.1 .4]$.) Since the elements of $L^{1}(G: A)$ are equivalence classes, if $f$ is an idempotent in $L^{1}(G: A)$, then we can assume that the representative satisfies $f(x)=\int_{G} f\left(x y^{-1}\right) f(y) d y$ for all $x \in G$. We make this assumption in the following theorem.

THeOREM 3. Let $f \in L^{1}(G: A)$ and suppose $\|f\|=1$ and $f * f=f$. Then $f$ is continuous and there exists a compact open subgroup $H$ of $G$ such that

(i) $f(x)=0, x \notin H$,

(ii) $f(x y)=\lambda(H) f(x) f(y), x, y \in H$,

(iii) $\|f(x)\|=(\lambda(H))^{-1}, x \in H$.

Proof.

and

$$
\|f(x)\| \leqslant \int_{G}\left\|f\left(x y^{-1}\right)\right\|\|f(x)\| d y
$$

$$
1=\int_{G}\|f(x)\| d x=\int_{G} \int_{G}\left\|f\left(x y^{-1}\right)\right\|\|f(y)\| d y d x
$$

so that

$$
\|f(x)\|=\int_{G}\left\|f\left(x y^{-1}\right)\right\|\|f(y)\| d y \quad \text { almost everywhere. }
$$

Hence if $\gamma(x)=\int_{G}\left\|f\left(x y^{-1}\right)\right\|\|f(y)\| d y$ then $\gamma$ is a norm one idempotent in $L^{1}(G)$. Hence there is a compact open subgroup $H$ such that $\gamma(x)$ $=\lambda(H)^{-1} \chi_{H}(x)$.

It follows that $\|f(x)\|=0$ if $x \notin H$, and that $\|f(x)\| \leqslant \lambda(H)^{-1}$ for all $x \in G$.

We may suppose $A$ has an identity and then identify $A$ as a subalgebra of $B(A)$. If we define for $x \in H$,

$$
T_{x} a=\lambda(H) f(x) a,
$$

then $\left\|T_{x}\right\| \leqslant 1$ and $\int_{H} T_{x y^{-1}} T_{y} d y=T_{x}$. By Theorem 2, $T_{x} T_{y}=T_{x y}$ and the result follows.

If $G$ is compact we may also consider the algebra $L^{p}(G: A)(1 \leqslant p<\infty)$ with the norm 


$$
\|f\|_{p}=\left\{\int\|j(x)\|^{p} d x\right\}^{1 / p} .
$$

Using a similar approach to that of [4] we obtain

THEOREM 4. If $f \in L^{p}(G: A)$ satisfies $\|f\|_{p}=1$ and $f * f=f$ then $f(x y)$ $=f(x) f(y)$ for all $x, y \in G$.

\section{REFERENCES}

1. F. P. Greenleaf, Norm decreasing homomorphisms of group algebras, Pacific J. Math. 15 (1965), 1187-1219. MR 33 \#3117.

2. E. Hille and R. S. Phillips, Functional analysis and semi-groups, rev. ed., Amer. Math. Soc. Colloq. Publ., vol. 31, Amer. Math. Soc., Providence, R. I., 1957. MR 19, 664.

3. W. Rudin and H. Schneider, Idempotents in group rings, Duke Math. J. 31 (1964), 586-602. MR 29 \#5119.

4. G. V. Wood, Homomorphisms of group algebras, Duke Math. J. 41 (1974), 255-261.

Department of Pure Mathematics, University College of Swansea, Swansea Sa2 8PP, WALEs, Great Britain 